\title{
Conhecimento sobre Cuidados Paliativos em Estudantes de Medicina da Universidade de Coimbra
}

\section{Knowledge of Palliative Care Among Medical Students of the University of Coimbra}

\author{
Sofia OLIVEIRA $\triangle^{1}$, Luiz Miguel SANTIAGO ${ }^{1}$, Marília DOURADO ${ }^{1}$ \\ Acta Med Port 2021 Apr;34(4):250-257 • https://doi.org/10.20344/amp.12590
}

\section{RESUMO}

Introdução: Cada vez mais doentes necessitam de cuidados paliativos. Os futuros médicos têm de estar preparados para esta realidade, pelo que é fundamental que desenvolvam competências ao longo do curso. Este estudo teve como objetivo avaliar o conhecimento sobre cuidados paliativos em estudantes de Medicina da Faculdade de Medicina da Universidade de Coimbra.

Material e Métodos: Foi realizado um estudo observacional e transversal, através da aplicação de um questionário a estudantes de Medicina, do quinto ano. Os dados recolhidos foram analisados no IBM ${ }^{\circledR}$ SPSS $^{\circledR}$ Statistics, versão 20 para Windows ${ }^{\circledR}$.

Resultados: Todos os 186 estudantes inquiridos mostraram saber o que são cuidados paliativos e reconheceram a sua importância. Do total, $52,7 \%$ afirmaram saber o que é um cuidador informal e 96,8\% discordaram que a prestação de cuidados paliativos apenas acontece em fim de vida; $88,2 \%$ não receberam formação para lidar com doentes terminais ou dependentes de cuidados permanentes. Somente $57,5 \%$ dizem ter recebido formação para comunicar 'más notícias', mas 70,4\% assumiram que não eram capazes de o fazer. Por outro lado, $89,8 \%$ revelaram não se sentir capacitados para cuidarem de doentes em cuidados paliativos e $98,4 \%$ manifestaram necessidade de mais formação nesta área.

Discussão: Com o número crescente de doentes a precisar de cuidados paliativos surge a necessidade de investigar se os estudantes de medicina estão a ser adequadamente preparados para cuidarem destes doentes e avaliar o seu grau de conhecimento no âmbito dos cuidados paliativos.

Conclusão: Os estudantes do quinto ano de Medicina sabem o que são cuidados paliativos e consideram-nos importantes. No entanto, não se sentem adequadamente preparados para cuidarem de doentes que precisam destes cuidados. É essencial refletir sobre como deverão ser formados os médicos e ponderar o plano de estudos do ensino médico, dada a falta de formação dos estudantes de medicina em cuidados paliativos.

Palavras-chave: Cuidados Paliativos; Educação Médica; Estudantes de Medicina; Inquéritos e Questionários; Portugal

\section{ABSTRACT}

Introduction: The need for palliative care is increasing. Future doctors must be prepared for this, so it is essential to develop skills during their undergraduate studies. The aim of this study was to evaluate the knowledge of medical students at the Faculty of Medicine of the University of Coimbra regarding palliative care.

Material and Methods: An observational and cross-sectional study was performed through the application of a questionnaire answered by fifth year medical students. The collected data were analyzed using IBM ${ }^{\circledR}$ SPSS $^{\circledR}$ Statistics, version 20 for Windows ${ }^{\circledR}$.

Results: All 186 students surveyed were aware of what palliative care is and recognized its importance. From the total, 52.7\% of students reported they knew what an informal caregiver is and $96.8 \%$ disagreed that palliative care is only provided at the end of life; $88.2 \%$ have never had any training on how to deal with palliative care patients. Only $57.5 \%$ of students reported that they had been trained to communicate 'bad news', but $70.4 \%$ assumed that they were not able to communicate such bad news. On the other hand, $89.8 \%$ reported their inability to take care of palliative patients and $98.4 \%$ admitted that they needed more training in this area.

Discussion: With the increasing number of patients who require palliative care, there is the need to investigate whether medical students are being adequately prepared to care for these patients and assess their knowledge level in this context.

Conclusion: Fifth-year medical students know what palliative care is and consider it important. However, they do not feel adequately trained and ready to take care of patients who need palliative care. It is essential to reflect on how physicians should be trained and to reconsider the medical syllabus, given the present lack of undergraduate medical training in palliative care.

Keywords: Education, Medical; Palliative Care; Portugal; Students, Medical; Surveys and Questionnaires

\section{INTRODUÇÃO}

Os cuidados paliativos (CP) assumem um papel cada vez mais importante na nossa sociedade, devido ao aumento da esperança média de vida e prevalência de doenças crónicas, ${ }^{1}$ consequentes das melhores condições de vida e cuidados de saúde, bem como da adoção de novos estilos de vida. ${ }^{2}$

Em Portugal, 70,7\% das mortes de adultos são por condições que beneficiariam de CP. ${ }^{3}$ Apesar da Lei de Bases dos Cuidados Paliativos (Lei n. ${ }^{\circ}$ 52/2012) ter assegurado o direito de acesso dos cidadãos portugueses a estes cuidados e criado a Rede Nacional de Cuidados Paliativos (RNCP), ${ }^{4}$ ainda existem défices e assimetrias de cobertura, entraves na referenciação e falta de recursos humanos. ${ }^{5}$ Além disso, o país quase não tem serviços de cuidados paliativos pediátricos (CPP) e as suas necessidades são crescentes. ${ }^{6} \mathrm{~A}$ formação dos profissionais também não corresponde à desejada e este parece ser o principal obstáculo para o sucesso das equipas de CP..$^{5,7}$

\footnotetext{
1. Faculdade de Medicina. Universidade de Coimbra. Coimbra. Portugal.

$\triangle$ Autor correspondente: Sofia Oliveira. sofia oliveira17@outlook.pt

Recebido: 22 de julho de 2019 - Aceite: 30 de março de 2020 - First published: 27 de agosto de 2020 - Online issue published: 01 de abril de 2021 Copyright $\odot$ Ordem dos Médicos 2021
} 
Segundo a Organização Mundial de Saúde (OMS), os CP visam "melhorar a qualidade de vida dos doentes, e suas famílias, que enfrentam problemas decorrentes de uma doença incurável com prognóstico limitado ou que ameaça a vida, através da prevenção e alívio do sofrimento, com recurso à identificação precoce, avaliação adequada e tratamento rigoroso dos problemas não só físicos, como a dor, mas também psicossociais e espirituais". ${ }^{8,9}$ Estes cuidados devem, portanto, ser assegurados por uma equipa interdisciplinar (constituída por médicos, enfermeiros e outros profissionais) com formação holística nas áreas que abrangem estes problemas. ${ }^{9}$ Neste sentido, o Conselho da Europa e a Comissão Nacional de Cuidados Paliativos (CNCP), tendo por base vários estudos, têm recomendado a promoção de estratégias de educação em CP.7,10,11 Em conformidade, a Resolução da Assembleia da República n. ${ }^{\circ}$ 5/2017, de 4 de janeiro, veio recomendar a implementação obrigatória de formação pré e pós-graduada em medicina paliativa e o seu reconhecimento como especialidade pela Ordem dos Médicos. ${ }^{12}$

Estudos internacionais evidenciam que o conhecimento dos estudantes de Medicina sobre CP é limitado e reforçam a importância de melhorar o ensino médico nesta área. ${ }^{13-18}$ Os futuros médicos revelam inseguranças face a problemas que dizem respeito a estes cuidados, particularmente a nível psicossocial e espiritual, mostrando que não estão preparados para cuidarem de doentes com doenças terminais e em fim de vida. ${ }^{13}$ As incertezas relacionadas com o controlo sintomático e a falta de à vontade para comunicar com estes doentes, são outras dificuldades apontadas pelos estudantes. ${ }^{14,15}$ Morais $^{19}$ veio igualmente mostrar que estudantes de vários cursos da área da saúde não têm conhecimento suficiente sobre $\mathrm{CP}$, nem estão devidamente capacitados para lidar com doentes que carecem destes cuidados.

O objetivo deste estudo é avaliar o conhecimento sobre CP em estudantes do Mestrado Integrado em Medicina (MIM) da Faculdade de Medicina da Universidade de Coimbra (FMUC).

\section{MATERIAL E MÉTODOS Tipo de estudo}

Observacional e transversal.

\section{População e amostra}

A população em estudo foi constituída por estudantes do quinto ano do MIM da FMUC $(n=330)$ a finalizar o ano letivo de 2017/2018, dado que este é o ano curricular que precede o estágio programado e orientado (EPO), sendo expectável que, até então, os estudantes tenham adquirido todas as competências teóricas para exercer em plenitude a prática clínica. Da população dos inscritos no quinto ano do MIM, foi estudada uma amostra aleatória pela aplicação do questionário aos presentes numa aula teórica obrigatória, em dia sorteado, na unidade curricular de Pediatria, com o único objetivo de reunir o máximo de estudantes e assim obter o maior número de respostas, no mais curto período de tempo. Apesar da obrigatoriedade existe limite de faltas às aulas, pelo que calculámos o tamanho da amostra de forma a assegurar a representatividade da população alvo, com um nível de confiança de $95 \%$ e uma margem de erro de $5 \%$, através de um instrumento de cálculo online, como sendo de $n=178 .{ }^{20}$

\section{Procedimentos prévios à recolha de dados}

Elaborámos um questionário com base noutros questionários, ${ }^{14,19}$ constituído por duas partes: uma respeitante à caracterização da população quanto ao sexo (variável nominal) e idade (variável quantitativa discreta), ambos critérios variáveis alvo de análise nesta investigação, e outra parte subdividida em duas secções, com itens de resposta 'Sim' e 'Não' e outras com opções de 'Discordo plenamente' (1) a 'Concordo plenamente' (4), numa escala do tipo Likert, perfazendo um total de 15 itens. Nesta segunda parte, são abordadas temáticas relacionadas com os $\mathrm{CP}$, de acordo com o objetivo deste estudo. Assim, com as questões colocadas pretendemos recolher informação sobre até que ponto sabem o que são os $\mathrm{CP}$, se frequentaram a Unidade Curricular de CP e Terapêutica da Dor, se já tinham sido cuidador informal, se já tinham frequentado alguma Unidade Curricular em que tivesse sido ensinado como fazer a transmissão de más notícias, se conheciam a definição de CP da Organização Mundial de Saúde, se tinham conhecimentos da existência de uma Rede Nacional de Cuidados Paliativos e se sentiam necessidade de ter mais informação sobre CP. O questionário continha ainda uma nota introdutória sobre o carácter voluntário e anónimo do seu preenchimento e solicitava seriedade nas respostas, tendo em conta a finalidade do mesmo.

A versão inicial do questionário foi submetida a um pré-teste, tendo sido aplicado a um conjunto de estudantes ( $\mathrm{n}$ = 20) para averiguar possíveis dificuldades na interpretação e/ou falhas na sua construção, tendo-se obtido, após pequenas alterações, a versão final.

\section{Recolha de dados}

Após informação prévia aos estudantes sobre o local e data da distribuição dos questionários, e com a autorização da professora regente, distribuíram-se os formulários durante uma aula teórica, e procedeu-se à sua recolha no final da sessão. Foi selecionada uma unidade curricular frequentada em simultâneo por quase todos os estudantes inscritos no quinto ano, o que assegurou o acesso ao maior número de estudantes possível.

\section{Análise estatística}

Analisaram-se os dados com recurso ao programa IBM ${ }^{\circledR}$ SPSS $^{\circledR}$ Statistics, versão 20 para Windows ${ }^{\circledR}$, após compilação e organização em base de dados Microsoft ${ }^{\circledR}$ Excel $^{\circledR}$ 2013. Recorreu-se a métodos de estatística descritiva, técnicas de análise inferencial e utilizou-se ainda os testes não paramétricos de Kolmogorov-Smirnov, $U$ de Mann-Whitney e $\chi^{2}$. Definiu-se como estatisticamente significativo o valor de $p<0,05$. Dado que para muitas perguntas as respostas 
Tabela 1 - Caracterização geral da amostra, segundo sexo e idade

\begin{tabular}{llcc}
\hline & & $\mathbf{n}$ & $\%$ \\
\hline \multirow{3}{*}{ Sexo } & Feminino & 133 & 71,5 \\
& Masculino & 53 & 28,5 \\
& Total & 186 & 100 \\
Idade & $<22$ anos & 2 & 1,1 \\
& $22-23$ anos & 127 & 68,3 \\
& $24-25$ anos & 46 & 24,7 \\
& $>25$ anos & 11 & 5,9 \\
\hline
\end{tabular}

n: número de indivíduos

extremas ficaram com escassos valores ou desertas de resultados, considerou-se, para efeitos de amostra e análise estatística, dois grupos de respostas 'concordo / concordo plenamente' e 'discordo / discordo plenamente'.

\section{Aspetos éticos}

O questionário distribuído aos estudantes continha uma nota introdutória com a apresentação do objetivo do estudo, esclarecimentos relativos ao seu preenchimento voluntário, garantia de que todos os resultados seriam anonimizados e solicitação de seriedade nas respostas, caso aceitasse responder. O projeto de trabalho foi submetido e aprovado pela Comissão de Ética da FMUC, e só executado após receção desta decisão.

\section{RESULTADOS}

Obtivemos uma amostra de 189 questionários, dos quais excluímos três por respostas incoerentes ou por duplas respostas. Refira-se que o tamanho calculado, previamente, para assegurar a representatividade da população em estudo foi de 178.

No total dos 186 indivíduos da amostra final - 56,4\% da população dos alunos do quinto ano - a maioria era do sexo feminino $(71,5 \%)$ (Tabela 1$)$. Verificou-se a não normalidade de distribuição da variável idade através do teste de Kolmogorov-Smirnov. A média de idades foi de 23,20 anos, sem diferença significativa entre sexos, $p=0,745$ ( $U$ Mann-Whitney), com uma mediana de 23 anos correspondendo ao quartil 25 a idade de 22 anos, e ao quartil 75 a de 24 anos.

Nas Tabelas 2 e 3 encontram-se descritos os resultados das respostas aos questionários. Todos os que responderam adequadamente $(98,4 \%)$ afirmam saber o que são CP

Tabela 2 - Resultados das respostas obtidas nas perguntas de 1 a 9

\begin{tabular}{|c|c|c|c|}
\hline & $\begin{array}{l}\operatorname{Sim} \\
\mathrm{n}(\%)\end{array}$ & $\begin{array}{l}\text { Não } \\
\text { n (\%) }\end{array}$ & Total \\
\hline P1. Sabe o que são Cuidados Paliativos? & $186(100)$ & 0 & 186 \\
\hline $\begin{array}{l}\text { P2. Frequentou ou está a frequentar a unidade curricular opcional de Cuidados Paliativos e } \\
\text { Terapêutica da Dor? }\end{array}$ & $21(11,3)$ & $165(88,7)$ & 186 \\
\hline P3. Sabe o que é um cuidador informal? & $98(52,7)$ & $88(47,3$ & 186 \\
\hline P4. Se respondeu sim à questão anterior, é ou já foi um cuidador informal? & $16(16,3)$ & $82(83,7)$ & 98 \\
\hline $\begin{array}{l}\text { P5. Recebeu formação específica, ao longo do curso, sobre como lidar com doentes em fase } \\
\text { terminal ou dependentes de cuidados permanentes? }\end{array}$ & $22(11,8)$ & $164(88,2)$ & 186 \\
\hline $\begin{array}{l}\text { P6. Recebeu formação específica, ao longo do curso, sobre como comunicar "más notícias" } \\
\text { aos doentes e familiares? }\end{array}$ & $107(57,5)$ & $79(42,5)$ & 186 \\
\hline $\begin{array}{l}\text { P7. Sabe se existe alguma definição da Organização Mundial de Saúde para Cuidados } \\
\text { Paliativos? }\end{array}$ & $84(45,2)$ & $102(54,8)$ & 186 \\
\hline P8. Sabe o que é a Rede Nacional de Cuidados Paliativos? & $99(53,2)$ & $87(46,8)$ & 186 \\
\hline P9. Sente necessidade de ter mais formação sobre Cuidados Paliativos? & $183(98,4)$ & $3(1,6)$ & 186 \\
\hline
\end{tabular}

Tabela 3 - Resultados das respostas obtidas nas afirmações de 10 a 15

\begin{tabular}{|c|c|c|c|c|c|c|}
\hline & & $\begin{array}{l}\text { Concordo } \\
\text { plenamente }\end{array}$ & Concordo & Discordo & $\begin{array}{c}\text { Discordo } \\
\text { plenamente }\end{array}$ & Total \\
\hline \multirow{2}{*}{$\begin{array}{l}\text { A10. Reconheço importância aos Cuidados Paliativos, } \\
\text { enquanto cuidados de saúde. }\end{array}$} & $\mathbf{n}$ & 172 & 14 & 0 & 0 & 186 \\
\hline & $\%$ & 92,5 & 7,5 & 0 & 0 & 100 \\
\hline \multirow{2}{*}{ A11. Os Cuidados Paliativos só têm início no fim de vida. } & $\mathbf{n}$ & 0 & 6 & 93 & 87 & 186 \\
\hline & $\%$ & 0 & 3,2 & 50 & 46,8 & 100 \\
\hline \multirow{2}{*}{$\begin{array}{l}\text { A12. Sinto-me capaz de lidar com doentes em fase } \\
\text { terminal ou dependentes de cuidados permanentes. }\end{array}$} & $\mathbf{n}$ & 2 & 17 & 130 & 37 & 186 \\
\hline & $\%$ & 1,1 & 9,1 & 69,9 & 19,9 & 100 \\
\hline \multirow{2}{*}{ A13. Sinto-me capacitado para comunicar "más notícias". } & $n$ & 0 & 55 & 102 & 29 & 186 \\
\hline & $\%$ & 0 & 29,6 & 54,8 & 15,6 & 100 \\
\hline \multirow{2}{*}{$\begin{array}{l}\text { A14. Sei quais são os fármacos usados no início de um } \\
\text { tratamento opióide. }\end{array}$} & $\mathbf{n}$ & 14 & 108 & 60 & 4 & 186 \\
\hline & $\%$ & 7,5 & 58,1 & 32,3 & 2,2 & 100 \\
\hline
\end{tabular}

n: número de indivíduos 
e reconhecem a sua importância. Quando questionados sobre se existe alguma definição da OMS para CP $(45,2 \%)$, se têm conhecimento do que é a $\operatorname{RNCP}(53,2 \%)$ ou se sabem o que é um cuidador informal $(52,7 \%)$, uma percentagem considerável dos indivíduos responde positivamente, afirmando saber do que se trata. Dos respondentes, 88,2\% dizem não ter recebido formação ao longo do curso para lidar com doentes em fase terminal ou dependentes de cuidados permanentes (Tabela 2) e 89,8\% (Tabela 3) não se sentem capazes de cuidar destes doentes. Pouco mais de metade dos alunos refere ter recebido formação para comunicar 'más notícias' (Tabela 2) e 70,4\% assumem não se sentir preparados para transmitir tais notícias a doentes e familiares, (Tabela 3 ). Para $98,4 \%$ há necessidade de mais formação sobre CP.

Procurámos avaliar se existiam diferenças estatisticamente significativas com base nas respostas por sexo às perguntas "Frequentou ou está a frequentar a unidade curricular opcional de Cuidados Paliativos e Terapêutica da Dor?" e "Se respondeu sim à questão anterior, é ou já foi um cuidador informal?".

Entre sexos, apenas se detetou uma diferença com significado estatístico na afirmação "Sinto-me capacitado para comunicar 'más notícias'”, em que $45,3 \%$ dos indivíduos do sexo masculino afirmaram que se sentem capacitados para comunicar 'más notícias', enquanto apenas $23,3 \%$ dos ele- mentos femininos assinalaram essa opção $(p=0,013)$

Dos estudantes que frequentaram ou estavam a frequentar a unidade curricular opcional de Cuidados Paliativos e Terapêutica da Dor (CPTD), $81 \%$ sabem da existência de uma definição da OMS para CP e 90,5\% asseguram saber o que é um cuidador informal. Todos têm conhecimento do que é a RNCP (Tabela 4). Entre estes estudantes, $42,9 \%$ referem ter recebido formação para lidar com doentes em fase terminal ou dependentes de cuidados permanentes, e sentem-se capazes de comunicar 'más notícias' (Tabela 4).

Depois dos estudantes responderem à pergunta "Sabe o que é um cuidador informal?" foi pedido que, se a resposta tivesse sido "Sim", respondessem à questão "Se respondeu sim à questão anterior, é ou já foi um cuidador informal?" a que maioritariamente responderam não: 83,7\% (Tabela 2). Foram verificadas diferenças estatisticamente significativas nas afirmações "Sinto-me capaz de lidar com doentes em fase terminal ou dependentes de cuidados permanentes" e "Sinto-me capacitado para comunicar 'más notícias"', com base na resposta anteriormente dada à questão "Se respondeu sim à questão anterior, é ou já foi um cuidador informal?" (Tabela 5 ).

É de $56,3 \%$ a proporção de alunos que foram ou eram cuidadores informais e assumiram sentirem-se capacitados para lidar com doentes em fase terminal ou dependentes

Tabela 4 - Resultados em função das respostas dadas à P2 "Frequentou ou está a frequentar a unidade curricular opcional de Cuidados Paliativos e Terapêutica da Dor?"

\begin{tabular}{|c|c|c|c|c|}
\hline & & \multicolumn{2}{|c|}{$\begin{array}{l}\text { Frequentou ou está a frequentar } \\
\text { a unidade curricular opcional de } \\
\text { Cuidados Paliativos e } \\
\text { Terapêutica da Dor? }\left(^{*}\right)\end{array}$} & \multirow[b]{2}{*}{$p$} \\
\hline & & $\begin{array}{c}\text { Sim } \\
\text { n (\%) }\end{array}$ & $\begin{array}{c}\text { Não } \\
\text { n (\%) }\end{array}$ & \\
\hline \multirow{3}{*}{ P3. Sabe o que é um cuidador informal? } & $\operatorname{Sim}$ & $19(90,5)$ & $79(47,9)$ & \multirow{3}{*}{$<0,001$} \\
\hline & Não & $2(9,5)$ & $86(52,1)$ & \\
\hline & Total & $21(100)$ & $165(100)$ & \\
\hline \multirow{3}{*}{$\begin{array}{l}\text { P5. Recebeu formação específica, ao longo do curso, sobre } \\
\text { como lidar com doentes em fase terminal ou dependentes de } \\
\text { cuidados permanentes? }\end{array}$} & Sim & $9(42,9)$ & $13(7,9)$ & \multirow{3}{*}{$<0,001$} \\
\hline & Não & $12(57,1)$ & $152(92,1)$ & \\
\hline & Total & $21(100)$ & $165(100)$ & \\
\hline \multirow{3}{*}{$\begin{array}{l}\text { P7. Sabe se existe alguma definição da Organização Mundial } \\
\text { de Saúde para Cuidados Paliativos? }\end{array}$} & $\operatorname{Sim}$ & $17(81)$ & $67(40,6)$ & \multirow{3}{*}{$<0,001$} \\
\hline & Não & $4(19)$ & $98(59,4)$ & \\
\hline & Total & $21(100)$ & $165(100)$ & \\
\hline \multirow{3}{*}{ P8. Sabe o que é a Rede Nacional de Cuidados Paliativos? } & Sim & $21(100)$ & $78(47,3)$ & \multirow{3}{*}{$<0,001$} \\
\hline & Não & $0(0)$ & $87(52,7)$ & \\
\hline & Total & $21(100)$ & $165(100)$ & \\
\hline \multirow{3}{*}{ A13. Sinto-me capacitado para comunicar "más notícias". } & $\begin{array}{l}\text { Concordo ou } \\
\text { Concordo } \\
\text { plenamente }\end{array}$ & $9(42,9)$ & $46(27,9)$ & \multirow{3}{*}{0,038} \\
\hline & $\begin{array}{l}\text { Discordo } \\
\text { ou discordo } \\
\text { plenamente }\end{array}$ & $12(57,1)$ & $119(72,1)$ & \\
\hline & Total & $21(100)$ & $165(100)$ & \\
\hline
\end{tabular}

n: número de indivíduos; $\left({ }^{*}\right) \chi^{2}$ 
Tabela 5 - Resultados em função das respostas dadas à P4 "Se respondeu sim à questão anterior, é ou já foi um cuidador informal?"

\begin{tabular}{|c|c|c|c|c|c|c|}
\hline & & \multicolumn{4}{|c|}{$\begin{array}{l}\text { P4. Se respondeu sim à questão } \\
\text { anterior, é ou já foi um cuidador } \\
\text { informal? }\end{array}$} & \multirow[b]{3}{*}{$p$} \\
\hline & & \multicolumn{2}{|c|}{ Sim } & \multicolumn{2}{|c|}{ Não } & \\
\hline & & $\mathbf{n}$ & $\%$ & $\mathbf{n}$ & $\%$ & \\
\hline \multirow{3}{*}{$\begin{array}{l}\text { A12. Sinto-me capaz de lidar com doentes } \\
\text { em fase terminal ou dependentes de cuidados } \\
\text { permanentes. }\end{array}$} & Concordo plenamente/ Concordo & 9 & 56,3 & 5 & 6,1 & \multirow{3}{*}{$<0,001$} \\
\hline & Discordo/ Discordo plenamente & 7 & 43,7 & 77 & 93,9 & \\
\hline & Total & 16 & 100 & 82 & 100 & \\
\hline \multirow{3}{*}{$\begin{array}{l}\text { A13. Sinto-me capacitado para comunicar } \\
\text { "más notícias". }\end{array}$} & Concordo plenamente/ Concordo & 10 & 62,5 & 23 & 28,0 & \multirow{3}{*}{0,006} \\
\hline & Discordo/ Discordo plenamente & 6 & 37,5 & 59 & 72,0 & \\
\hline & Total & 16 & 100 & 82 & 100 & \\
\hline
\end{tabular}

n: número de indivíduos; $(*) \chi^{2}$

de cuidados permanentes, e $62,5 \%$ mostram-se preparados para comunicar 'más notícias'. Por fim, as respostas às perguntas "Sinto-me capaz de lidar com doentes em fase terminal ou dependentes de cuidados permanentes", "Sinto-me capacitado para comunicar "más notícias" e "Sei quais são os fármacos usados no início de um tratamento opióide" sugere-nos que os estudantes têm a perceção de conhecer os fármacos e de saber quais os procedimentos técnicos a usar. Contudo, não se sentem capazes, ou habilitados, para acompanhar, na prática, doentes em CP.

\section{DISCUSSÃO}

Eram objetivos deste trabalho avaliar o conhecimento dos alunos do MIM da FMUC quanto a conhecimentos sobre vários aspetos relativos aos $\mathrm{CP}$ numa fase adiantada da formação, em que se espera que tenham adquirido todas as competências teóricas para exercer a prática clínica. As respostas foram obtidas num curto período de tempo para evitar contaminações de respostas que seriam possíveis com outras formas de aplicação do questionário. Os dados foram tratados para ser quantitativamente possível obter resultados sólidos que pudessem fundamentar o interesse e a necessidade de desenvolver o ensino nesta área de atuação médica.

Os nossos resultados mostram que os estudantes do quinto ano do MIM da FMUC referem saber o que são CP e reconhecem a sua importância enquanto cuidados de saúde. Estes resultados vão ao encontro dos de Eyigor, ${ }^{15}$ que também verificou que os estudantes com grau de formação semelhante sabem o que são CP e são capazes de os definir. $O$ estudo de Morais, ${ }^{19}$ realizado com estudantes de áreas científicas da Saúde, mostra igualmente que os estudantes estão familiarizados com o conceito de $\mathrm{CP}$ e que os consideram importantes no contexto da prestação de cuidados de saúde, e para a sociedade no geral. No nosso estudo, os estudantes, além de lhes reconhecerem o interesse, foram ainda capazes de identificar que os CP podem ser iniciados precocemente e não só no fim de vida. Contudo, outras questões e conceitos, direta ou indiretamente relacionados com a temática dos $\mathrm{CP}$, como o de cuidador informal, ou a RNCP, são desconhecidos por $52,7 \%$ e $53,2 \%$, respetivamente, dos estudantes incluídos na amostra, deixando transparecer que estes assuntos são pouco ou nada abordados durante o curso.

$\mathrm{Na}$ generalidade, os estudantes inquiridos não se sentem capacitados para lidar com doentes terminais ou dependentes de cuidados permanentes, particularmente em $\mathrm{CP}$, apesar de conhecerem bem os fundamentos teóricos, os fármacos a usar, por exemplo para o controlo da dor, e outros procedimentos técnicos. Resultados muito idênticos foram identificados no trabalho de Jolien Pieters et al, ${ }^{21} \mathrm{o}$ que deve fazer-nos refletir acerca da necessidade de ajustar os modelos pedagógicos à prática médica, tornando-os mais adequados à formação de médicos capazes de lidar com as situações de grande sofrimento e dependência e, muitas vezes, de fim de vida - situações cada vez mais comuns e complexas na realidade da nossa população. Embora o objetivo primário seja sempre a formação de médicos com excelentes conhecimentos científicos, deve promover-se também a aquisição de competências e de gestos clínicos, e incentivar a aprendizagem de valores profissionais e éticos, para que possam ser sempre bons médicos, independentemente do lugar ou dos doentes que tenham de tratar. Por isso, parece-nos essencial uma adequação curricular do ensino pré-graduado, que passe por um ensino interprofissional, orientado para a reflexão e a resolução dos problemas, com a integração de 'módulos' que visem estimular competências (o saber e o saber-fazer) e preparem os futuros médicos para a prática clínica centrada no doente. Neste sentido, a inclusão de uma unidade curricular de Cuidados Paliativos/Medicina Paliativa, obrigatória em todos os planos curriculares nos cursos de Mestrado Integrado em Medicina, seria uma mais valia, contribuindo para a melhor formação dos futuros médicos através do desenvolvimento de um conjunto de capacidades de comunicação, comportamentos, competências éticas e morais indispensáveis a uma relação médico/doente/ família aberta, pautada pelo respeito, empatia e compaixão, valores essenciais nesta área da Medicina. ${ }^{22-27}$

Em resumo, o ensino deve passar de uma vertente meramente teórica para outra eminentemente prática, em que o aluno aprende a fazer a translação do conhecimento para a atuação no doente.

Por outro lado, os estudantes também não se sentem 
devidamente preparados, ou capazes, para comunicar 'más notícias', o que pode ser reflexo da falta de formação nestas áreas durante o curso. Corroborando estes resultados, Pinheiro ${ }^{14}$ reporta que grande parte dos estudantes de Medicina do quinto e sexto ano não conhece a definição da OMS para CP, nem recebeu formação para cuidar de doentes terminais e/ou comunicar 'más notícias' a doentes e familiares. Os estudos de Weber et al, ${ }^{13}$ Eyigor $^{15}$ e Morais ${ }^{19}$ reforçam a falta de preparação dos estudantes para cuidar de doentes que necessitam de CP, e salientam as dificuldades de comunicação com estes doentes. Pode-se, pois, afirmar que os resultados deste estudo vão ao encontro dos de outros autores, e reforçam a ideia de que a formação dos estudantes de medicina nesta área não é a desejável.

Considerando a evolução consistente da esperança de vida global, o consequente envelhecimento da população, e os rápidos avanços da Medicina, que cada vez mais possibilitam a cura da doença ou a sua transformação em doença crónica, ${ }^{28}$ parece ser necessário investir na formação de jovens médicos nesta área do cuidado, capacitando-os para fazer face a este tipo de necessidades e desafios que, ao que tudo indica, serão tendencialmente maiores no futuro. É precisamente neste sentido que têm sido emitidas as recomendações internacionais e nacionais. ${ }^{7,29-31}$

Quanto às diferenças entre sexos, verificou-se que os indivíduos do sexo masculino se sentem mais capazes de comunicar 'más notícias', resultados para os quais não encontrámos, na literatura, uma explicação adequada. Tanto mais que, contrariamente, Orlander et $a^{\beta 2}$ não encontraram diferenças entre os sexos relativamente a quão preparados os indivíduos se sentem para esta tarefa. Ainda no que se refere às competências comunicacionais, verificou-se que a confiança para comunicar 'más notícias' foi mais elevada e significativa em quem frequentou, ou estava a frequentar, a disciplina de CPTD e em quem foi, ou era, cuidador informal.

Quem frequentou, ou estava a frequentar, a unidade curricular de CPTD detém mais conhecimentos que os restantes, sobretudo teóricos. Recebeu também mais formação para lidar com doentes em fase terminal, ou dependentes de cuidados permanentes. No entanto, poucos estudantes (menos de um terço relativamente ao número total de estudantes no mesmo ano curricular) frequentaram essa unidade curricular. Num estudo efetuado por Centeno et a/33 foi também demonstrado que os estudantes que frequentaram um curso de CP referiram que o curso os preparou para a prática clínica e que o que aprenderam, além de útil, era aplicável a qualquer tipo de doente. Ao mesmo tempo, mostraram-se surpreendidos com a visão humana e holística que o curso lhes providenciou.

No nosso estudo, os estudantes que foram ou eram cuidadores informais parecem dominar melhor os conhecimentos práticos, pois sentem-se mais capacitados para cuidar de doentes que carecem de CP. Estes resultados são compreensíveis e podem ser explicados pelo conhecimento e pela prática que tiveram de adquirir e desenvol- ver, como cuidadores. Resultados idênticos, reforçando os nossos, foram descritos por Eyigor ${ }^{15}$ e Anderson et al, ${ }^{34}$ que também constataram que os estudantes cuidadores de doentes com necessidades paliativas detinham mais conhecimentos em CP do que aqueles que não acompanharam doentes com aquelas necessidades.

Parece ser claro que investir no ensino dos CP é fundamental para desenvolver as diferentes competências necessárias ao acompanhamento e tratamento adequado dos doentes com indicação para CP, o que, como já foi dito anteriormente, é uma realidade atual de tendência crescente.

\section{Pontos fortes e limitações}

Apesar do questionário ser anónimo e, por isso, assegurar uma boa fiabilidade de respostas, não é possível garantir que estas correspondam verdadeiramente à realidade. O preenchimento do questionário foi voluntário, pelo que não se obteve uma amostra aleatória simples, o que poderá constituir uma fonte de erro. Para evitar respostas neutras, foi utilizada uma escala do tipo Likert com quatro itens de resposta ao invés do formato típico com cinco itens, o que, segundo Lozano et $a l,{ }^{35}$ é igualmente válido e assegura a confiabilidade das respostas.

A metodologia usada permitiu investigar o objetivo deste trabalho e foi semelhante à de outros estudos. ${ }^{14,15}$ Contudo, poder-se-ia ter aplicado o questionário a outros anos do MIM ou até mesmo realizado um estudo longitudinal, avaliando a mesma amostra ao longo do curso. Um desenho longitudinal com medições ao longo dos anos permitirá acompanhar a evolução do conhecimento, que vai sendo adquirido ao longo da formação pré-graduada. Um tal estudo identificaria, ao longo do período de formação, qual a forma mais adequada de organizar a as várias unidades curriculares para obter os melhores resultados em termos de integração de conhecimentos em Cuidados Paliativos.

\section{CONCLUSÃO}

O nosso estudo permite concluir que os estudantes do quinto ano do MIM da FMUC sabem o que são CP e reconhecem a sua importância, mas não estão, no entanto, adequadamente preparados para cuidar de doentes que necessitam destes cuidados.

Aqueles que frequentaram, ou estavam a frequentar, a unidade curricular de CPTD têm um maior conhecimento que os restantes, e encontram-se mais capacitados para lidar com estes doentes, tal como se esperava. O mesmo se verificou em quem foi, ou era, cuidador informal.

Este estudo vem advertir sobre a necessidade de remodelar o plano de estudos e integrar de forma apropriada e obrigatória o ensino dos CP na formação médica pré-graduada, dada a desadequada preparação dos futuros médicos e o número crescente de doentes que precisam de $\mathrm{CP}$. É fundamental investir mais nesta área de ensino, tanto a nível teórico como prático, para que seja possível mudar o atual paradigma.

No futuro, seria importante alargar o estudo a estudantes de outros anos do MIM, para avaliar a evolução dos 
conhecimentos adquiridos sobre os CP ao longo da sua formação. Seria também importante realizar estudos que permitissem identificar as características que apoiam o facto dos indivíduos do sexo masculino se sentirem mais capazes de comunicar 'más notícias', mesmo sem formação específica para isso.

\section{PROTEÇÃO DE PESSOAS E ANIMAIS}

Os autores declaram que os procedimentos seguidos estavam de acordo com os regulamentos estabelecidos pelos responsáveis da Comissão de Investigação Clínica e Ética e de acordo com a Declaração de Helsínquia da Associação Médica Mundial emitida em 2013.

\section{REFERÊNCIAS}

1. World Palliative Care Alliance, World Health Organization. Global atlas of Palliative Care at the End of Life [e-book]. London: World Palliative Care Alliance; 2014. [consultado 2018 nov 24]. Disponível em: https:// www.who.int/nmh/Global_Atlas_of_Palliative_Care.pdf.

2. Ministério da Saúde. Retrato da Saúde 2018 [e-book]. Portugal: Ministério da Saúde; 2018. [consultado 2018 Nov 24]. Disponível em: https://www.sns.gov.pt/retrato-da-saude-2018/.

3. Gomes B, Pinheiro MJ, Lopes S, Brito M, Sarmento VP, Ferreira PL, et al. Risk factors for hospital death in conditions needing palliative care: nationwide population-based death certificate study. Palliat Med. 2018;32:891-901.

4. Portugal. Decreto-Lei $n^{\circ}$ 52/2012. Diário da República, I Série, n. ${ }^{\circ} 172$ (2012/09/05). p.5119-24. I

5. Observatório Português dos Sistemas de Saúde. Relatório Primavera 2017 - Viver em tempos incertos: sustentabilidade e equidade na saúde [e-book]. 2017. [consultado 2018 nov 24]. Disponível em: https://opss. $\mathrm{pt} /$ relatorios/relatorio-primavera-2017/.

6. Lacerda AF, Gomes B. Trends in cause and place of death for children in Portugal (a European country with no Paediatric palliative care) during 1987-2011: a population-based study. BMC Pediatr. 2017;17:215.

7. Comissão Nacional de Cuidados Paliativos. Plano Estratégico para o Desenvolvimento dos Cuidados Paliativos Biénio 2017-2018 [e-book]. 2016. [consultado 2018 nov 24]. Disponível em: https://www. sns.gov.pt/wp-content/uploads/2016/09/Plano-Estrat\%C3\%A9gico CP 2017-2018-1-1.pdf.

8. World Health Organization. Definition of Palliative Care. Geneva: WHO; 2019. [consultado 2018 nov 24]. Disponível em: http://www.who.int/ cancer/palliative/definition/en/.

9. Associação Nacional de Cuidados Paliativos. Organização de Serviços em Cuidados Paliativos - Recomendações da ANCP [e-book]. Porto: 2006. [consultado 2018 nov 24]. Disponível em: https://www.apcp.com. pt/uploads/Recomendacoes_Organizacao de Servicos.pdf.

10. Council of Europe. Recommendation Rec (2003) 24 of the Committee of Ministers to Member States on the Organisation of Palliative Care [e-book]. Strasbourg; 2003. [consultado 2018 nov 24]. Disponível em: https://www.coe.int/t/dg3/health/Source/Rec(2003)24 en.pdf.

11. Pereira SM, Hernández-Marrero P, Silva AJ. Os cuidados paliativos na formação conducente ao exercício de profissões na área da saúde em Portugal. Lisboa: Observatório Português dos Cuidados Paliativos: 2016. [consultado 2018 nov 24]. Disponível em: https://ics.lisboa.ucp. pt/pt-pt/system/files/assets/files/os-cp-na-formacao-pre-graduada-emsaude.pdf.

12. Portugal. Resolução da Assembleia da República n. ${ }^{\circ}$ 5/2017. Diário da República, I Série, n. ${ }^{\circ} 3(2017 / 01 / 04)$. p.28.

13. Weber M, Schmiedel S, Nauck F, Alt-Epping B. Knowledge and attitude of final - year medical students in Germany towards palliative care an interinstitutional questionnaire-based study. BMC Palliat Care. 2011;10:19.

14. Pinheiro TR. Avaliação do grau de conhecimento sobre cuidados paliativos e dor dos estudantes de medicina do quinto e sextos anos. Mundo Saude. 2010;34:320-6.

15. Eyigor S. Fifth-year medical students' knowledge of palliative care and their views on the subject. J Palliat Med. 2013;8:941-6.

16. Lemos CF, Barros GS, Melo NC, Amorim FF, Santana AN. Avaliação do conhecimento em cuidados paliativos em estudantes durante o curso de Medicina. Rev Bras Educ Med. 2017;41:278-82.

\section{CONFIDENCIALIDADE DOS DADOS}

Os autores declaram ter seguido os protocolos do seu centro de trabalho acerca da publicação de dados.

\section{CONFLITOS DE INTERESSE}

Os autores declaram não ter conflitos de interesses relacionados com o presente trabalho.

\section{FONTES DE FINANCIAMENTO}

Este trabalho não recebeu qualquer tipo de suporte financeiro de nenhuma entidade no domínio público ou privado.

17. Hesselink BA, Pasman HR, Wal G, Soethout MB, Onwuteaka-Philipsen BD. Education on end-of-life care in the medical curriculum: students' opinions and knowledge. J Palliat Med. 2010;4:381-7.

18. Pandey S, Gaire D, Dhakal S, Jaishwal N, Kharal PM, Vaidya P. Perception of palliative care among medical students in a teaching hospital. JNMA J Nepal Med Assoc. 2015;53:113-7.

19. Morais AM. Cuidados Continuados e Paliativos (CCP): um estudo descritivo sobre o conhecimento de estudantes de áreas da saúde. Tese de mestrado em Psicologia Clínica e da Saúde. Gandra: Instituto Superior de Ciências da Saúde do Norte; 2013.

20. Santos GE. Cálculo amostral: calculadora on-line [homepage na Internet]. [consultado 2018 maio 11]. Disponível em: http://www. calculoamostral.vai.la,

21. Pieters J, Dolmans DH, Verstegen DM, Warmenhoven FC, Courtens $\mathrm{AM}$, van den Beuken-van Everdingen $\mathrm{MH}$. Palliative care education in the undergraduate medical curricula: students' views on the importance of, their confidence in, and knowledge of palliative care. BMC Palliative Care. 2019;18:72

22. Martins e Silva J. Educação médica e profissionalismo. Acta Med Port. 2013;26:420-7.

23. General Medical Council. Tomorrow's Doctors. Recommendations on Undergraduate Medical Education. London; 2003. [consultado 2019 nov 29]. Disponível em: https://www.educacionmedica.net , pdf , tomorrowdoc.

24. Cohen JJ. Medical education in an era of health-care reform. Perspect Biol Med. 2011;54:61-7

25. Hinchey KT, Rothberg MB. Can residents learn to be good doctors without harming patients? J Gen Intern Med. 2010;25:760-1.

26. Humphrey HJ, Levinson D, Smith L. The medical school on the university campus: $20^{\text {th }}$ century legacy and $21^{\text {st }}$ century aspirations. Acad Med. 2010;85:273-82.

27. Comissão Nacional de Cuidados Paliativos (CNCP). Plano Estratégico para o Desenvolvimento dos Cuidados Paliativos - Biénio 20192020. Lisboa: Ministério da Saúde; 2019. [consultado 2019 nov 29]. Disponível em:_https://www.sns.gov.pt > PEDCP-2019-2020-versaofinal-10.02.2019.pdf.

28. Neto IG. Cuidados paliativos: princípios e conceitos fundamentais. In Barbosa A, Pina PR, Tavares F, Neto IG, editores. Manual de Cuidados Paliativos. $3^{a}$ ed. Lisboa: Núcleo de Cuidados Paliativos/Centro de Bioética/Faculdade de Medicina da Universidade de Lisboa; 2016. p.122.

29. European Association for Palliative Care. Curriculum in palliative care for undergraduate medical education [e-book]. 2007. [consultado 2019 jan 5]. Disponível em: https://www.dgpalliativmedizin.de/images/stories/ EAPC Curriculum for Undergraduate Medical Education.pdf

30. World Heath Organization - Europe. Les déterminants sociaux de la Santé: Les Faits [e-book]. 2004. [consultado 2019 jan 5] Disponível em: http://www.euro.who.int/_data/assets/pdf_file/0006/98439/E82519.pdf.

31. European Federation of Older Persons. Making palliative care a priority topic on the European Health agenda: and recommendations for the development of palliative care in Europe [e-book]. Graz, Austria; 2004. [consultado 2019 jan 5]. Disponível em: https://www.apcp.com.pt/ uploads/Cons.Europa_EURAG_PalliativeCareProject_2004.pdf.

32. Orlander JD, Fincke BG, Hermanns D, Johnson GA. Medical residents' first clearly remembered experiences of giving bad news. J Gen Intern Med. 2002;17:825-31. 
33. Centeno C, Ballesteros M, Carrasco JM, Arantzamendi M. Does palliative care education matter to medical students? The experience of attending an undergraduate course in palliative care. BMJ Support Palliat Care. 2016;6:128-38.

34. Anderson WG, Williams JE, Bost JE, Barnard D. Exposure to death is associated with positive attitudes and higher knowledge about end-oflife care in graduating medical students. J Palliat Med. 2008;9:1227-33.

35. Lozano LM, García-Cueto E, Muñiz J. Effect of the number of response categories on the reliability and validity of rating scales. Methodology. 2008;4:73-9. 